This item was submitted to Loughborough's Research Repository by the author.

Items in Figshare are protected by copyright, with all rights reserved, unless otherwise indicated.

\title{
Innovations towards the next generation of shallow flow models
}

PLEASE CITE THE PUBLISHED VERSION

https://doi.org/10.1016/j.advwatres.2021.103867

PUBLISHER

Elsevier BV

VERSION

AM (Accepted Manuscript)

PUBLISHER STATEMENT

This paper was accepted for publication in the journal Advances in Water Resources and the definitive published version is available at https://doi.org/10.1016/j.advwatres.2021.103867

\section{LICENCE}

CC BY-NC-ND 4.0

\section{REPOSITORY RECORD}

Özgen-Xian, Ilhan, Xilin Xia, Qiuhua Liang, Reinhard Hinkelmann, Dongfang Liang, and Jingming Hou. 2021. "Innovations Towards the Next Generation of Shallow Flow Models". Loughborough University.

https://hdl.handle.net/2134/13718161.v1. 


\section{Innovations Towards the Next Generation of Shallow Flow Models}

Ilhan Özgen-Xian ${ }^{1}, X_{i l i n} X_{i a}^{2,{ }^{*}}$, Qiuhua Liang ${ }^{2}$, Reinhard Hinkelmann ${ }^{3}$, Dongfang Liang ${ }^{4}$, Jingming $\mathrm{Hou}^{5}$

1 Lawrence Berkeley National Laboratory, 1 Cyclotron Road, M/S 74R316C, Berkeley, CA 94720, USA

2 School of Architecture, Building and Civil Engineering, Loughborough University, Loughborough, Leicestershire, LE11 3TU, UK

3 Chair of Water Resources Management and Modeling of Hydrosystems, Technische Universität Berlin, Berlin, Germany

4 Department of Engineering, University of Cambridge, Cambridge, CB2 1PZ, UK

5 State Key Laboratory of Eco-hydraulics in Northwest Arid Region of China, Xi'an University of Technology, Xi'an, China

Many types of environmental and geophysical flows occur on a horizontal scale that is much larger than the vertical scale. Examples include river flows, overland flows, and granular avalanches. These flows may be described using the depth-integrated equations, which are derived by integrating the three-dimensional governing equations, for example the Navier-Stokes equations, along the vertical direction. Shallow flow models, which solve these depth-integrated equations, are widely used for both research and practical applications, including understanding catchment processes and predicting hydrological hazards such as flooding.

The last two decades saw great advances in developing robust shallow flow models for real world applications. Robust numerical schemes have been developed to handle wet/dry interfaces, stiff source terms, and equilibrium solutions in real world applications. However, there still exist prevailing challenges, such as insufficient computational efficiency and limited capability in describing coupled physics, which have restricted their current applications to either small-domain, coarse resolution, or single-process problems.

These challenges are particularly relevant with the simulation of natural hazards brought by extreme weather events such as flooding. To better understand and predict the impacts of extreme weather events, models need to operate in much larger domains (e.g., cities or catchments) at sufficiently fine resolutions (e.g., resolving buildings) and represent interactive physical processes by further solving coupled equations. As the magnitude and frequency of extreme weather events and the following natural hazards rapidly increase due to climate change, a new generation of shallow flow models providing such capabilities is urgently needed to inform

\footnotetext{
* Corresponding author, email: x.xia2@lboro.ac.uk
} 
the development of more effective hazard risk reduction strategies to better protect people and their properties.

Advances in Water Resources is a key publication venue for research at the frontier of shallow flow model development, with a broad readership covering both researchers and practitioners. This special issue is an outcome of a collection of research efforts initiated in the session Development and application of the next generation of shallow flow models organised as part of the 13th International Conference on Hydroinformatics, held in Palermo, Italy on July 1-6, 2018. During the preparation of the special issue, submissions from the broader research community were encouraged to better represent the state of the research and highlight the current and future challenges in this field.

A total of 17 papers are collected, which broadly address three interwoven research challenges: (1) robust treatment of complex domain topography, wet/dry interfaces, and stiff source terms, especially for high-order schemes; (2) improvement of computational efficiency by using highperformance computing or multiscale modelling techniques for large-scale applications; and (3) development of rigorous numerical methods to solve coupled equations for better prediction of multiple physical processes.

High-order numerical schemes, particularly the discontinuous Galerkin (DG) schemes, is gaining popularity in the shallow flow research community because they can potentially achieve similar simulation accuracy using coarser grid size in comparison with finite volume models, hence reducing simulation time. In addition, a DG scheme achieves high-order accuracy using small and local stencils, which allows for a more efficient parallelisation. However, DG schemes introduce additional degrees of freedom to obtain this high-order accuracy, which inevitably increases computational cost. Faghih-Naini et al. (2020) aim to address this and increase the computational efficiency via a novel quadrature-free DG scheme on block-structured triangular meshes. Another approach for increasing the computational efficiency is the use of adaptive mesh refinement (AMR). Kesserwani et al. (2019) and Kesserwani \& Sharifian (2020) present a multiwavelet-based AMR strategy, which in combination with a DG scheme yields a robust and efficient shallow flow model. The multiwavelet-based AMR strategy for DG schemes is also explored in CaviedesVoullième et al. (2020) in field-scale application cases. The multiwavelet-based AMR makes the DG scheme a competitive alternative to the more established second-order finite volume schemes. While second-order accuracy is usually considered to be sufficient for environmental flow modelling, certain applications may require even higher order accuracy. Navas-Montilla \& Juez (2020) use a high-order WENO-ADER scheme to capture turbulent structures in a channel. Without the high-order accuracy, the numerical diffusion will smear out the steep gradients and hence the small turbulent eddies.

The challenge of the high computational cost of shallow flow models can also be addressed by using modern GPUs to accelerate the computation. Dazzi et al. (2020) present a GPUaccelerated shallow flow model with internal boundary conditions to represent urban structures such as bridges. Echeverribar et al. (2019) and Xia et al. (2019) use GPU-accelerated shallow flow models for large scale flood inundation modelling applications, e.g. simulating flooding from source (rainfall) to impact (inundation) in a $2500 \mathrm{~km}^{2}$ catchment. 
Many physical processes involve more than one scale, that is to say multiscale problems, which may be approached by means of multiscale modelling methods, also aiming to increase computational efficiency. In addition to the AMR methods that have already been mentioned (Caviedes-Voullième et al., 2020; Kesserwani et al., 2019; Kesserwani and Sharifian, 2020), other multiscale approaches, for example subgrid-based methods, have also been developed. For example, Dazzi et al. (2020) use internal boundaries to account for subgrid-scale urban features. Li \& Hodges (2020) use an artificial porosity approach to account for subgrid-scale structures in narrow channels. The artificial porosity approach is also considered by Ferrari et al. (2020), who present a Riemann solver for the porous shallow flow equations. The Riemann problem for the porous shallow flow equations is mathematically similar to the Riemann problem of a shallow flow across a width discontinuity. Valiani \& Caleffi (2019) analyse the augmented shallow flow system that results from this problem and derive a Riemann solver. In this special issue, we also have La Rocca et al. (2020) presenting a Lattice-Boltzmann method for shallow granular flows. The Lattice-Boltzmann methods result directly from a multiscale consideration of the underlying physics and have presented great potential in efficient shallow flow modelling.

Process coupling has been an active topic in shallow water modelling, as water flow drives many other processes in both rural and urban environments. Many shallow flow models expand their capabilities to include these relevant processes to tackle specific science and engineering problems such as multi-hazard modelling. The coupling of multiple processes increases the complexity of model formulation and implementation, and may impact the robustness of the simulation. Fernández-Pato et al. (2020) present a coupled surface-subsurface flow model in a study involving morphodynamics. The interface between surface and subsurface creates stiff terms in the governing equations and the morphodynamics introduces additional nonlinearity to the model solution. González-Aguirre et al. (2020) present a robust model for bedload sediment transport in shallow flows using a path-conservative approach. Krvavica (2020) studies the computational efficiency of first-order accurate numerical schemes for solving the two-layer shallow flow equations. Li et al. (2020) present a novel method to couple the one-dimensional and two-dimensional approaches to efficiently simulate pipe flows in drainage systems.

A potential direction for future research in shallow flow modelling can be found in (Shaw et al., 2020), which derives wavelet-based stochastic approaches to model uncertainty. Instead of using the conventional Monte Carlo methods to run many simulations, the new method solves the equations only once and directly uses the probability distributions as variables. This method has the potential to enable much more efficient quantification of uncertainty in various applications such as probabilistic flood forecasting and risk assessment.

The collection of papers in this special issue well represents the current research forefront towards the next generation shallow flow models. The advances in computing technologies and numerical methods have provided some unprecedented opportunities to develop shallow flow models for new applications, such as catchment scale hydrological processes and city scale flood routing. The emerging capabilities will enable us to better understand and model in more detail the hydrodynamic processes at different scales. This then provides the essential tools and information to address pressing societal needs, such as informing the development of effective 
risk management strategies for hydrological hazards and hence increasing societal resilience to climate change to sustain socio-economic development.

\section{Acknowledgments}

We thank the local organizing committee of the 13th International Conference on Hydroinformatics, held on July 1-6, 2018 in Palermo, Italy, for providing the opportunity for organising the session that resulted in this special issue. We specially thank the journal's editorin-chief Prof G. Sander for handling this special issue.

\section{References}

Caviedes-Voullième, D., Gerhard, N., Sikstel, A., Müller, S., 2020. Multiwavelet-based mesh adaptivity with Discontinuous Galerkin schemes: Exploring 2D shallow water problems. Adv. Water Resour. 138. https://doi.org/10.1016/j.advwatres.2020.103559

Dazzi, S., Vacondio, R., Mignosa, P., 2020. Internal boundary conditions for a GPU-accelerated 2D shallow water model: Implementation and applications. Adv. Water Resour. 137, 103525. https://doi.org/10.1016/j.advwatres.2020.103525

Echeverribar, I., Morales-Hernández, M., Brufau, P., García-Navarro, P., 2019. 2D numerical simulation of unsteady flows for large scale floods prediction in real time. Adv. Water Resour. 134. https://doi.org/10.1016/j.advwatres.2019.103444

Faghih-Naini, S., Kuckuk, S., Aizinger, V., Zint, D., Grosso, R., Köstler, H., 2020. Quadraturefree discontinuous Galerkin method with code generation features for shallow water equations on automatically generated block-structured meshes. Adv. Water Resour. 138, 103552. https://doi.org/10.1016/j.advwatres.2020.103552

Fernández-Pato, J., Martínez-Aranda, S., García-Navarro, P., 2020. A 2D finite volume simulation tool to enable the assessment of combined hydrological and morphodynamical processes in mountain catchments. Adv. Water Resour. 141. https://doi.org/10.1016/j.advwatres.2020.103617

Ferrari, A., Vacondio, R., Mignosa, P., 2020. A second-order numerical scheme for the porous shallow water equations based on a DOT ADER augmented Riemann solver. Adv. Water Resour. 140, 103587. https://doi.org/10.1016/j.advwatres.2020.103587

González-Aguirre, J.C., Castro, M.J., Morales de Luna, T., 2020. A robust model for rapidly varying flows over movable bottom with suspended and bedload transport: Modelling and numerical approach. Adv. Water Resour. 140. https://doi.org/10.1016/j.advwatres.2020.103575

Kesserwani, G., Sharifian, M.K., 2020. (Multi)wavelets increase both accuracy and efficiency of standard Godunov-type hydrodynamic models: Robust 2D approaches. Adv. Water Resour. 144, 103693. https://doi.org/10.1016/j.advwatres.2020.103693

Kesserwani, G., Shaw, J., Sharifian, M.K., Bau, D., Keylock, C.J., Bates, P.D., Ryan, J.K., 2019. (Multi)wavelets increase both accuracy and efficiency of standard Godunov-type hydrodynamic models. Adv. Water Resour. 129, 31-55.

https://doi.org/10.1016/j.advwatres.2019.04.019 
Krvavica, N., 2020. Re-evaluating efficiency of first-order numerical schemes for two-layer shallow water systems by considering different eigenvalue solutions. Adv. Water Resour. 137. https://doi.org/10.1016/j.advwatres.2020.103508

La Rocca, M., Prestininzi, P., Elango, L., Hinkelmann, R., Montessori, A., 2020. Depth averaged modelling of loose rectangular granular piles collapsing in water. Adv. Water Resour. 143, 103663. https://doi.org/10.1016/j.advwatres.2020.103663

Li, Q., Liang, Q., Xia, X., 2020. A novel 1D-2D coupled model for hydrodynamic simulation of flows in drainage networks. Adv. Water Resour. 137. https://doi.org/10.1016/j.advwatres.2020.103519

Li, Z., Hodges, B.R., 2020. On modeling subgrid-scale macro-structures in narrow twisted channels. Adv. Water Resour. 135, 103465. https://doi.org/10.1016/j.advwatres.2019.103465

Navas-Montilla, A., Juez, C., 2020. Numerical ability of hyperbolic flux solvers to compute 2D shear layers in turbulent shallow flows. Adv. Water Resour. 135, 103482. https://doi.org/10.1016/j.advwatres.2019.103482

Shaw, J., Kesserwani, G., Pettersson, P., 2020. Probabilistic Godunov-type hydrodynamic modelling under multiple uncertainties: robust wavelet-based formulations. Adv. Water Resour. 137, 103526. https://doi.org/10.1016/j.advwatres.2020.103526

Valiani, A., Caleffi, V., 2019. Dam break in rectangular channels with different upstreamdownstream widths. Adv. Water Resour. 132. https://doi.org/10.1016/j.advwatres.2019.103389

Xia, X., Liang, Q., Ming, X., 2019. A full-scale fluvial flood modelling framework based on a highperformance integrated hydrodynamic modelling system ( HiPIMS ). Adv. Water Resour. 132, 103392. https://doi.org/10.1016/j.advwatres.2019.103392 